\title{
Dual-focus narrow band imaging for the detection of intestinal metaplasia and atrophic gastritis
}

Gastric cancer is one of the most common neoplastic diseases in developed Western countries, and is characterized by a poor prognosis when diagnosed at a late stage [1]. Atrophic gastritis and gastric intestinal metaplasia (GIM) are well-known risk factors for the development of gastric cancer. For this reason, endoscopy with multiple biopsies is recommended in patients with atrophic gastritis or GIM, to exclude preneoplastic or neoplastic tissue [1]. Magnifying endoscopy with narrow band imaging (NBI) has been shown to be reliable for in vivo diagnosis of atrophic gastritis and GIM [1-4]. However, while magnifying endoscopy is routinely used by Asian endoscopists, it is not established in most European and Northern American endoscopy centers. Recently, a novel endoscope with NBI and dual-focus capability has been introduced to improve the quality of endoscopic imaging [5]. Dual focus allows the user to select between two focus settings by pushing a button on the scope. This results in an optimized close view of the tissue up to an 80 -fold optical magnification. To the best of our knowledge, no reports exist so far on the use of this new technique for the detection and characterization of gastric lesions. We report a case in which dual-focus narrow band imaging was used to diagnose atrophic gastritis with intestinal metaplasia and obtain targeted biopsies in the same session.

A 57-year-old woman with dyspeptic symptoms was referred for esophagogastroduodenoscopy. Physical examination was unremarkable and laboratory parameters were within reference ranges. Highdefinition white-light endoscopy (Olympus, Tokyo, Japan) showed extensive absence of gastric rugae and visible vessels upon the gastric mucosa, consistent with macroscopic signs of atrophic gastritis ( $\bullet$ Fig. 1). Additionally, NBI revealed the presence of blue-whitish patchy areas on the distal corpus ( $\bullet$ Fig. 2 ). Dual-focus NBI revealed isolated flat lesions up to $2 \mathrm{~mm}$ in diameter with a subtle villous-like morphology and the appearance of light blue crests on the epithelial surface ( Fig.3). Accordingly, an in vivo diagnosis of atrophic gastritis with intestinal metaplasia was made and targeted biopsies were obtained, which confirmed the in vivo diagnosis of atrophic gastritis with foci of intestinal metaplasia.

This case is interesting as it demonstrates for the first time the potential of dual-focus NBI for diagnosis of early gastric lesions. The potential benefits of this new technique are that in vivo diagnosis of suspect lesions could be performed in real time without any time delay, and that targeted biopsies could be performed for subsequent histopathological analysis, thereby allowing the assignment of proper surveillance intervals. As dual focus is combined with an automatic algorithm (Prefreeze) which selects the sharpest image when the image is frozen by the user, high-magnification imaging becomes feasible even for nonexpert users. Larger trials on the potential of this technique for early diagnosis and surveillance of patients with early gastric lesions are now warranted.

Endoscopy_UCTN_Code_CCL_1AB_2AD_3AB

\section{Competing interests: None}

\section{Gian Eugenio Tontini ${ }^{1,2}$, Annette Lindner ${ }^{1}$, Michael Vieth ${ }^{3}$, Maurizio Vecchi $^{2,4}$, Markus F. Neurath ${ }^{1}$, Helmut Neumann'}

${ }^{1}$ Department of Medicine I, University of Erlangen-Nuremberg, Germany

${ }^{2}$ Gastroenterology and Digestive Endoscopy Unit, IRCCS Policlinico San Donato, San Donato Milanese, Italy

${ }^{3}$ Institute of Pathology, Klinikum Bayreuth, Bayreuth, Germany

${ }^{4}$ Department of Biomedical Science

for Health, University of Milan, Italy

\section{Acknowledgments $\nabla$}

Gian Eugenio Tontini has a grant from the Italian Group for the study of Irritable Bowel Disease (IG-IBD) supporting his research work at the Department of Medicine I, University of Erlangen-Nuremberg.

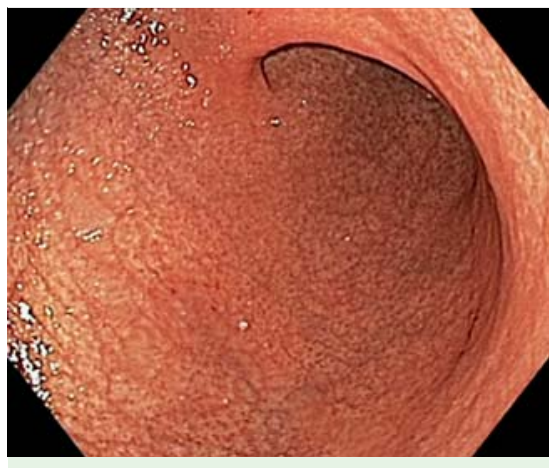

Fig. 1 High-definition white-light endoscopy showing extensive absence of gastric rugae and visible vessels upon the gastric mucosa, consistent with macroscopic signs of atrophic gastritis.

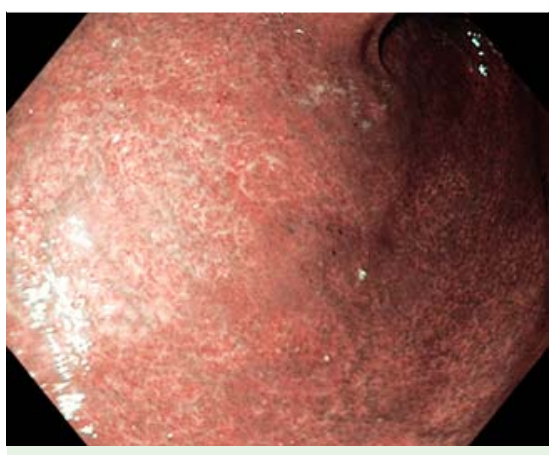

Fig. 2 Narrow band imaging of gastric mucosa depicting blue-whitish patchy areas on the distal corpus.

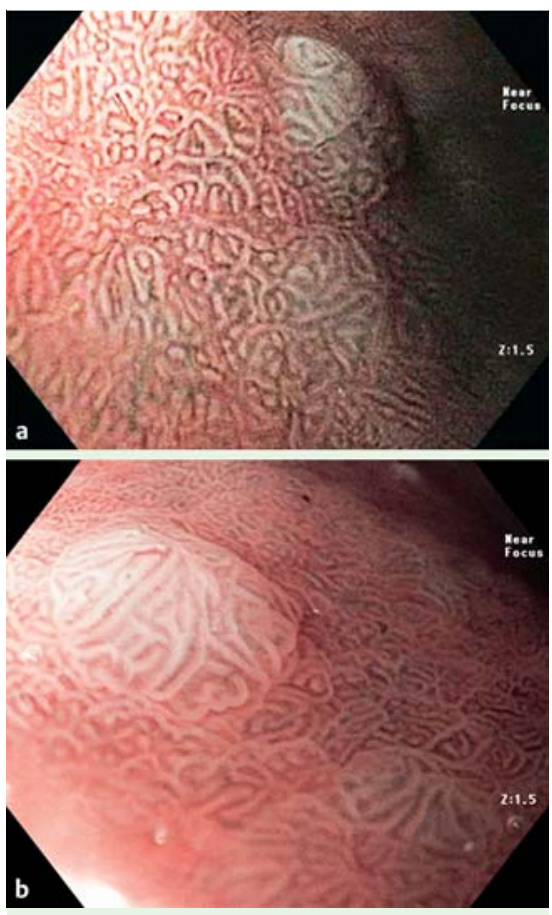

Fig. 3 a, b High-magnification dual-focus narrow band imaging of isolated flat lesions $(2 \mathrm{~mm}$ diameter) with a subtle villous-like morphology and the appearance of light blue crests. 


\section{References}

1 Dinis-Ribeiro M, Areia M, de Vries AC et al. Management of precancerous conditions and lesions in the stomach (MAPS): guideline from the European Society of Gastrointestinal Endoscopy (ESGE), European Helicobacter Study Group (EHSG), European Society of Pathology (ESP), and the Sociedade Portuguesa de Endoscopia Digestiva (SPED). Endoscopy 2012; 44: 74-94

2 Savarino E, Corbo M, Dulbecco $P$ et al. Narrow-band imaging with magnifying endoscopy is accurate for detecting gastric intestinal metaplasia. World J Gastroenterol 2013; 19: $2668-2675$
3 Uedo N, Ishihara R, Iishi $H$ et al. A new method of diagnosing gastric intestinal metaplasia: narrow-band imaging with magnifying endoscopy. Endoscopy 2006; 38: 819-824

4 Neumann H, Mönkemüller K, Neurath MF et al. Advanced endoscopic imaging. Diagn Ther Endosc 2013; 2013: 206839

5 Singh R, Shahzad MA, Tam $W$ et al. Preliminary feasibility study using a novel narrowband imaging system with dual focus magnification capability in Barrett's esophagus: is the time ripe to abandon random biopsies? Dig Endosc 2013; 25 (Suppl. 02): $151-156$

\section{Bibliography}

Dol http://dx.doi.org/

10.1055/s-0033-1359121

Endoscopy 2014; 46: E47-E48

(c) Georg Thieme Verlag KG

Stuttgart · New York

ISSN 0013-726X

\section{Corresponding author}

\section{Gian Eugenio Tontini, MD, PhD}

Department of Medicine I

University of Erlangen-Nuremberg

Ulmenweg 18

91054 Erlangen

Germany

Fax: +49-9131-8535209

gianeugeniotontini@libero.it

gianeugeniotontini@gmail.com 